\title{
Emergencia y Trayecto de las Arterias Epigástricas Superiores: Aplicación en los Abcesos Quirúrgicos Abdominales
}

\author{
Emergency and Trajectory of the Superior Epigastric Arteries: \\ Aplication on the Assessement Abdominal Surgery \\ "Amauri Clemente da Rocha; **Luiz Ferreira de Souza; ${ }^{* * *}$ Célio Fernando de Sousa-Rodrigues; \\ ${ }^{* * * * *}$ Benedito Herani Filho; ${ }^{* * * * *}$ Mario Jorge Jucá \& ${ }^{* * * * * * *}$ Alexandre Magno Nunes
}

ROCHA, A. C.; SOUZA, L. F.; SOUSA RODRIGUES, C. F.; HERANI FILHO, B.; JUCÁ, M. J. \& NUNES, A. M. Emergencia y trayecto de las arterias epigástricas superiores: Aplicación en los abcesos quirúrgicos abdominales. Int. J. Morphol., 24(1):25-30, 2006.

RESUMEN: El conocimiento de la vascularización de la pared anterior del abdomen es un importante instrumento para la realización de incisiones y punciones abdominales, contribuyendo a evitar algunas complicaciones reurrentes de este procedimiento. Con el propósito de estudiar la emergencia y el trayecto de las arterias epigástricas superiores, fueron disecados 32 cadáveres adultos, del sexo masculino, no fijados, blancos y no blancos, con edades entre 18 y 65 años. Se observó el trayecto de las arterias epigástricas superiores, su emergencia y su forma de distribución. Las arterias epigástricas superiores emergieron por atrás del $7^{\circ}$ cartílago costal en todos los casos. En el $75 \%$ de los casos las arterias epigástricas se presentaron como un tronco único y en 15 casos (23,44\%) bifurcadas, siendo 11 a la derecha y 4 a la izquierda y en 1 caso $(1,56 \%)$ a la izquierda. En este último, caso dio origen a tres ramos principales. Cuando era un tronco único, su trayecto fue descendente de medial para lateral en relación al margen lateral del músculo recto del abdomen. Cuando era bifurcada, sus ramos se separaron uno del otro luego de su emergencia, volviendo a convergir próximo a la cicatriz umbilical.

PALABRAS CLAVE: Arterias epigástricas; Pared abdominal; Punción abdominal.

\section{INTRODUCCIÓN}

Las vías de accesos quirúrgicas convencionales, aún con restauración adecuada de las estructuras parietales, causan disfunciones que pueden cursar con gran morbilidad (Rubinstein, 1999).

La abundante vascularización de la pared anterior del abdomen representa uno de los grandes factores para las lesiones vasculares en cirugías abdominales (Hurd et al., 1994).

Las arterias epigástricas superiores son vasos importantes de la pared anterior del abdomen, siendo el conocimiento de su anatomía muy importante en las incisiones y punciones abdominales, por el riesgo de lesión de estos vasos durante los procedimientos. Sin embargo, existen controversias en relación a la forma de distribución de estos vasos (Rocha, 2005).
Elbaz et al. (1975); Basmajian(1980); Gardner et al. (1982); Castro (1985); Gray \& Goss (1988); Schaefer (1989); Latarjet \& Ruiz-Liard (1993); Meunier et al. (1997); Dangelo \& Fattini (1998) y Rubinstein, describieron a la arteria epigástrica superior como un ramo de la arteria torácica interna, que emerge en el abdomen posterior al $7^{\circ}$ cartílago costal, por la cara posterior del músculo recto del abdomen y dentro de su vaina. Sin embargo, Briand et al. (1999) citaron su emergencia, posterior al $6^{\circ}$ cartílago costal.

Gerard (1912), Tandler (1929); Paturet (1951); Voss \& Herrlinger (1964); Hollinshead (1966); Haddad (1968); Elbaz et al.; Nahai et al. (1976); Romanes (1976); Meunier et al.; Basmajian; Gardner et al.; Castro; Gray \& Goss; Schaeffer; Latarjet \& Ruiz-Liard; Dangelo \& Fattini y Rubinstein describieron que la arteria epigástrica superior

* Mg. Cs. Prof. auxiliar de Anatomía humana de la Universidad Federal de Alagoas y Universidad de Ciencias de la Salud de Alagoas, Maceió, Brasil.

** Prof. Mg. Cs., Ph.D. M.D. Anatomía Humana, Universidad Federal de Alagoas, Maceió, Brasil.

**** Prof. Mg. Cs., Ph.D. M.D. Anatomía Humana, Universidad Federal de Alagoas y Universidad de Ciencias de la Salud de Alagoas, Maceió, Brasil.

***** Prof. Mg. Cs., Ph.D. M.D. Gastroenterología Quirúrgica Universidad Federal de São Paulo - Escuela Paulista de Medicina, São Paulo, Brasil.

****** Prof. Mg. Cs., Ph.D. M.D. Disciplina de Coloproctología de la Universidad Federal de Alagoas, Maceió, Brasil.

****** Alumno de Medicina de la Universidad de Ciencias de la Salud de Alagoas, Maceió, Brasil. 
penetraba en la pared anterior del abdomen como un tronco único, irrigando al músculo recto del abdomen, tejido subcutáneo, proceso xifoides, diafragma, además de dar ramos para el ligamento falciforme del hígado.

En un estudio anatómico, a través de examen contrastado con sulfato de bario, de los vasos de la pared anterior del abdomen, O'dey et al. (2004) describieron un tronco principal de las arterias epigástricas superiores, con división en dos ramos: uno lateral y otro media, que se anastomosan con las arterias intercostales más inferiores.

Milloy et al. (1960) disecaron 100 músculos rectos del abdomen y observaron que en el $54 \%$ de los casos las arterias epigástricas superiores emergían en un tronco único en nivel del ángulo infra-esternal y en un 38\% emergían en la pared anterior del abdomen, dividiéndose en dos ramos: uno lateral y otro medial. En tres casos estaban ausentes.

Durante las disecciones anatómicas de la pared anterior del abdomen y debido a las discordancias encontradas en la literatura, nos propusimos estudiar la emergencia y el trayecto de las arterias epigástricas superiores, con el objetivo de aportar nuevos conocimientos anatómicos, los cuales posibiliten disminuir el riesgo de lesión de estos vasos durante las incisiones quirúrgicas y punciones en la pared superior del abdomen.

\section{MATERIAL Y MÉTODO}

Fueron disecados 32 cadáveres, adultos, de sexo masculino, sin raza definida y no fijados, con edades entre 18 y 65 años, cedidos por el Laboratorio de Anatomía Humana de la Universidad Federal de Alagoas y Universidad de Ciencias de la Salud de Alagoas. Cada cadáver fue colocado en posición decúbito dorsal y se le efectuó una incisión biacromio-esterno-xifo-pubiana, siendo rebatida la piel y el tejido celular subcutáneo, para exposición de la vaina del músculo recto del abdomen. Luego, fue retrirada la vaina del músculo recto del abdomen y realizada la desección de la arteria epigástrica superior, siendo observadas la emergencia, número de ramos principales y trayecto de esta arteria y de sus ramos terminales.

\section{RESULTADOS}

Las arterias epigástricas superiores estuvieron presentes en un $100 \%$ de los casos, emergiendo en todos ellos por detrás del $7^{\circ}$ cartílago costal.
Con respecto a la forma de distribución, en 48 casos (75\%) se presentó como tronco único (Fig. 1), en 15 casos $(23,44 \%)$ de forma bifurcada, constituyendo dos ramos principales: uno lateral y otro medial (Fig. 2), siendo 11 casos a la derecha y 4 casos a la izquierda; y en sólo un caso en el lado izquierdo $(1,56 \%)$, esta arteria se presentó con tres ramos principales (Fig. 3).

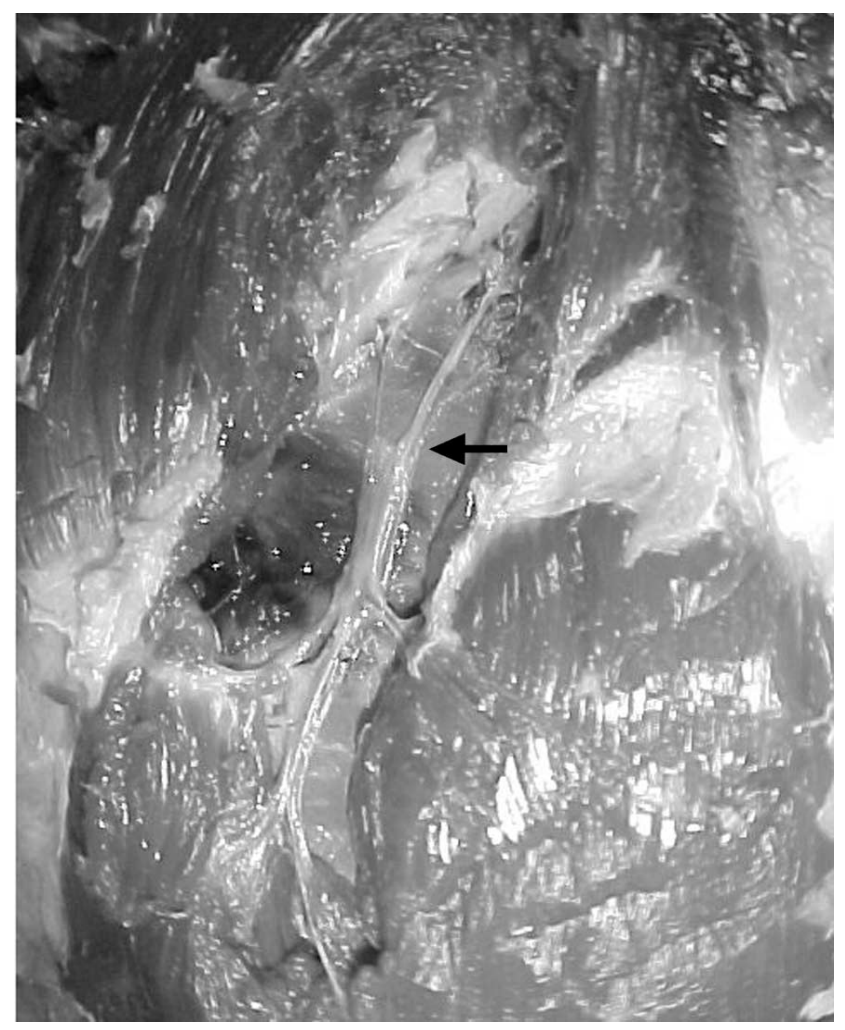

Fig. 1. Emergencia de la arteria epigástrica superior de forma troncular.

Con relación al trayecto de las arterias epigástricas superiores, en los casos en que emergieron en tronco único, se presentaron con un trayecto descendente, inicialmente próximo a la línea mediana y, enseguida, alejándose de esta línea en dirección al margen lateral del músculo recto del abdomen, principalmente a la derecha. En los casos de bifurcación, estos ramos se alejaron uno del otro, volviendo a converger próximo al ombligo.

Sólo en el lado izquierdo, el ramo lateral constituyó la continuación de la arteria epigástrica superior en los cuatro casos de bifurcación.

Fue observada en cinco casos, a la derecha y en dos casos a la izquierda, la presencia de un ramo oriundo de la arteria epigástrica superior que se dirigió lateral y paralelamente al margen costal y, posteriormente, siguió un trayecto descendente paralelo al margen lateral del músculo recto del abdomen (Fig. 4). 


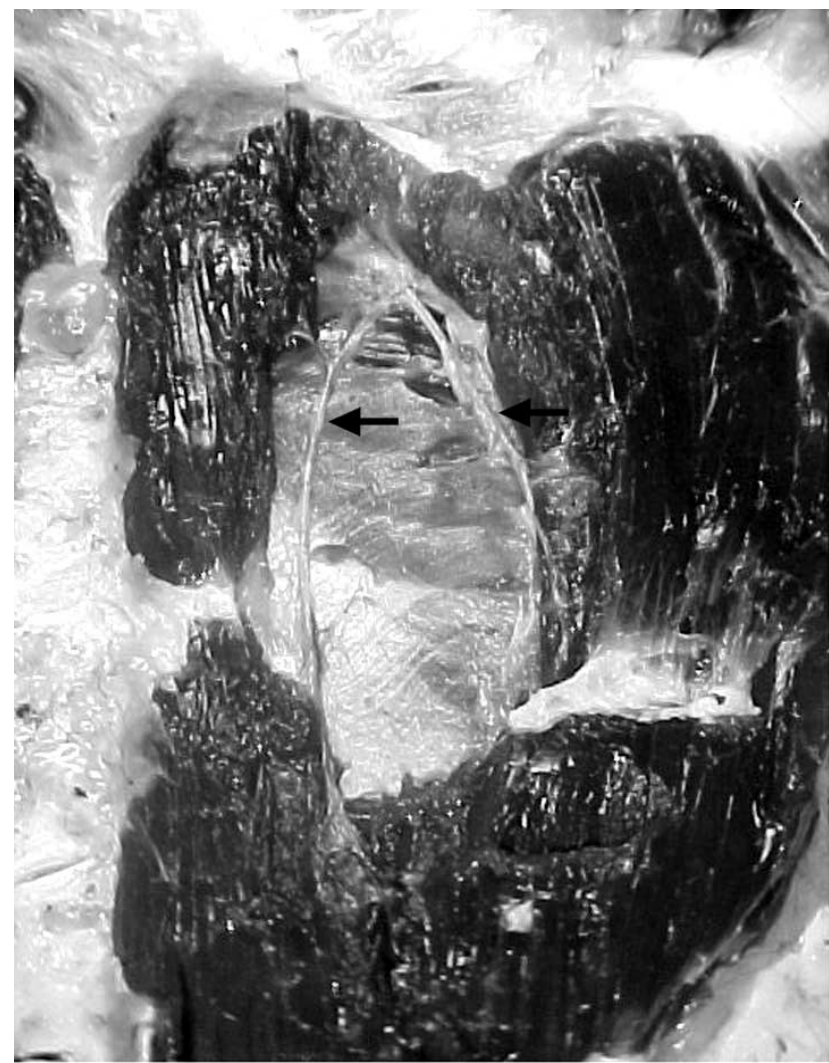

Fig. 2. Forma bifurcada de la arteria epigástrica superior.

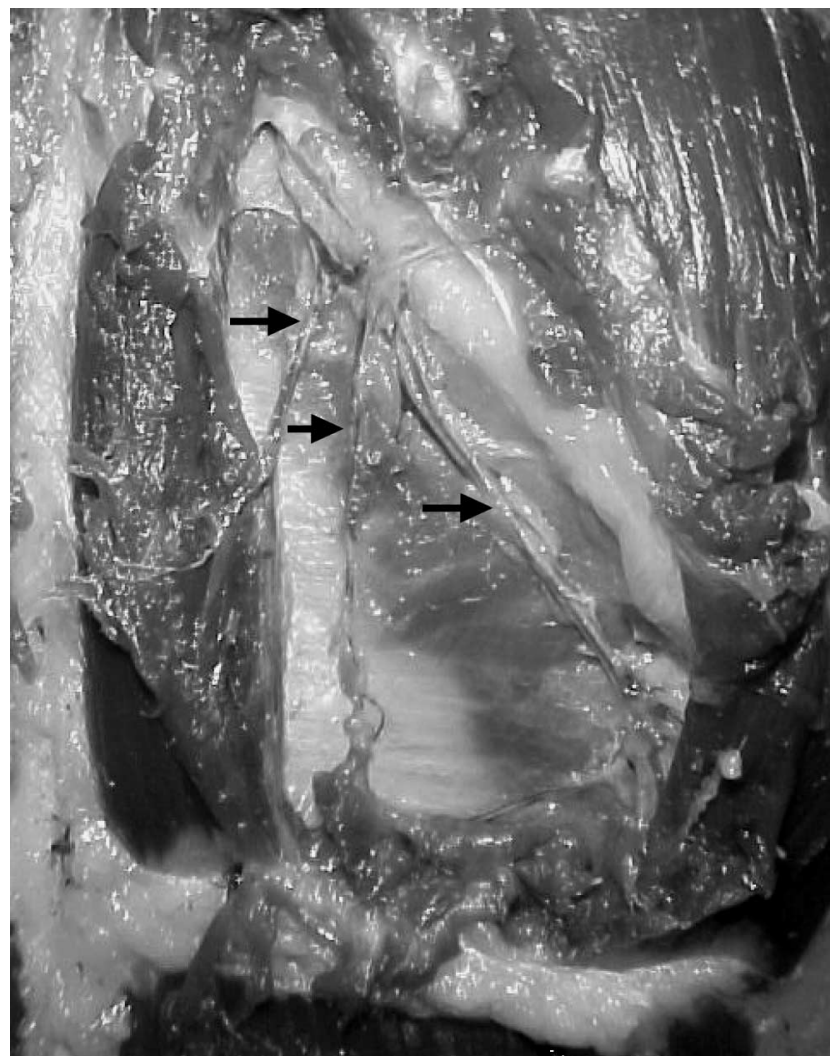

Fig. 3. Forma trifurcada de la arteria epigástrica superior.

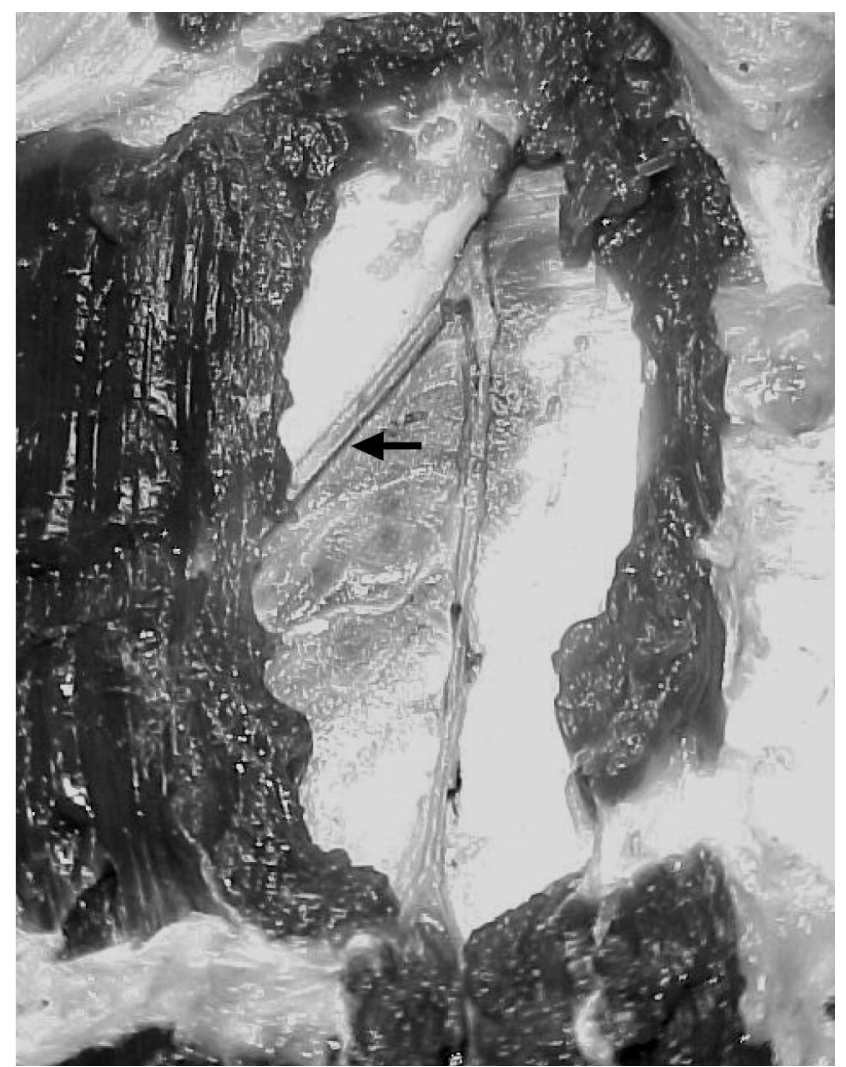

Fig. 4. Ramo de la arteria epigástrica superior paralela al margen costal.

\section{DISCUSIÓN}

Durante mucho tiempo, las arterias epigástricas superiores no fueron descritas con detalles anatómicos, hasta las citaciones realizadas por Gerard.

El conocimiento de la topografía de los vasos epigástricos superiores se hace de extrema necesidad para los cirujanos, en la tentativa de disminuir los riesgos de lesionarlos en las incisiones abdominales como fue descrito por Orts (1970) y Rocha (2005).

La emergencia de las arterias epigástricas superiores por detrás del $7^{\circ}$ cartílago costal, dentro de la vaina del músculo recto del abdomen, como fue descrita por Gerard, Tandler; Paturet; Voss \& Herrlinger; Hollinshead; Elbaz et al.; Basmajian; Gardner et al.; Castro; Gray \& Goss; Schaefer; Latarjet \& Ruiz-Liard; Meunier; Dangelo \& Fattini y Rubinstein, es semejante a los resultados obtenidos en este estudio, pero difieren de las descripciones de Briand et al., quienes señalaron la emergencia de estas arterias detrás de la $6^{a}$ costilla. 
En las disecciones realizadas por Milloy et al., la arteria epigástrica superior emergía hacia la pared anterior del abdomen a nivel del ángulo infra-esternal en un 54\% de los casos y estaban ausentes en el 3\% de los casos. Esto último, no fue verificado. Así, la ausencia de las arterias epigástricas superiores es un hallazgo extremadamente raro, encontrándose pocas citaciones en la literatura (Rocha).

Cuando la arteria epigástrica superior se dirige hacia la pared anterior del abdomen, define su trayecto dependiendo si es única o se divide en dos o más ramos principales, como lo describieron Rouviére (1943); Boyd et al. (1984); Briand et al. En esta investigación, fue observada una ramificación que varió de dos a tres ramos, esta última manera no ha sido descrita en la literatura.

En este estudio, la arteria epigástrica superior emergió hacia la pared abdominal a través del trígono esternocostal, lo que ya ha sido verificado por Tandler; Paturet; Testut \& Latarjet (1959); Voss \& Herrlinger; Hollinshead; Basmajian; Gardner et al.; Castro; Gray \& Goss; Schaefer; Moore (1992); Latarjet \& Ruiz-Liard; Rubinstein y Rocha. Para los autores señalados, la arteria epigástrica superior penetra en la pared anterior del abdomen, por detrás del músculo recto del abdomen dentro de su vaina, teniendo un trayecto descendente, de lateral para medial, irrigando estos músculos y la piel suprayacente, hecho confirmado en nuestros hallazgos.

Testut \& Latarjet; Gardner et al.; Gray \& Goss; Snell (1995) y Rubinstein describieron que desde la arteria epigástrica superior se originan ramos musculares y cutáneos, y pequeños ramos que siguen el ligamento falciforme, los cuales se anastomosan con la arteria hepática y un pequeño ramo que pasa al frente del proceso xifoides, para servir de circulación colateral entre las arterias epigástrica superiores. Esta distribución no fue evidenciada en las disecciones del estudio, ya que estos ramos son estremadamente delgados, rompiéndose con facilidad, como señalan Boyd et al.

Observamos que las arterias epigástricas superiores disminuían de calibre muy rápidamente en la mayoría de los casos, durante su transcurso daban origen a pequeños ramos secundarios, que se distribuyeron en la musculatura de la pa- red abdominal, hecho verificado por Boyd et al.; Por otra parte, Lindner (1989), describe ramos provenientes de las arterias epigástricas superiores, que se anastomosan con las tres últimas arterias intercostales anteriores y con ramos de las arterias lumbares altas, caso no encontrado en la literatura.

Con respecto a la forma de ramificación de la arteria epigástrica superior Tandler; Haddad; Nahai et al.; Elbaz et al. $y$ Shaeffer describen que este vaso entra en la pared anterior del abdomen en un tronco único, dando ramos para los tejidos adyacentes. Según Milloy et al. esta forma de emergencia ocurrió en un 54\% de 97 arterias epigástricas superiores disecadas, patrón también observado en nuestro estudio, donde en 48 casos (un 75\%) de 64 arterias disecadas, demostraba ser la forma más común de distribución.

Arnold (1972) describió un ramo paralelo al margen costal originado de la arteria epigástrica superior, el cual denominó arteria costomarginal, que se dividía en dos ramos terminales: uno lateral y otro medial, siendo este último la continuación de la arteria epigástrica superior. Esto también fue descrito por Elbaz et al., pero, en nuestro estudio, esta manera de ramificación sólo fue evidenciada en 4 casos, siendo su ramo lateral la continuación en el lado izquierdo.

Moon \& Taylor (1988) estudiando las arterias epigástricas superiores en 64 cadáveres no fijados, a través de examen contrastado, encontraron diferentes patrones de distribución. Así, en el 57\% de las casos identificaron un tronco único dividiéndose en ramos lateral y medial y en $14 \%$ identificaron varios ramos haciendo anastomosis entre sí, muy diferente de la forma de distribución descrita por la mayoría de los autores y no observada en este estudio.

Meunier et al. estudiando, a través de fluxometria con Doppler color, el aporte sanguíneo de colgajos miocutáneos de la pared anterior del abdomen, observaron en 20 pacientes mastectomizadas, que las arterias epigástricas superiores se dividían en dos ramos terminales, las cuales seguían un trayecto paralelo al eje longitudinal de este músculo, semejante a la forma observada en el $23,44 \%$ de nuestros casos, caracterizándose este patrón de presentación como una variación anatómica según lo señalado por Boyd et al.

ROCHA, A.C.; SOUZA, L.F.; SOUSA-RODRIGUES, C.F.; FILHO, B.H.; JUCÁ, M.J. \& NUNES, A.M. Emergency and trajectory of the superior epigastric arteries: Aplication on the assessement abdominal surgery. Int. J. Morphol., 24(1):25-30, 2006.

SUMMARY: The knowledge of the vascularization of the anterior abdominal wall is very important on the incisions and abdominal punctures, contributing to avoid the injuries of this vessels during this procedures. With the goal to study the emergency and the trajectory of the superior epigastric arteries, 32 adult not preserved cadavers, of the masculine sex, white and not white, with age between 18 and 65 years were studied. The trajectory of the superior epigastric arteries, your emergency and distribution form were observed. The superior epigástric arteries emerged behind to 7 th costal cartilage in all the cases. In $75 \%$ of the cases they appeared like an only trunk and in 15 cases $(23,44 \%)$ they appeared forked, being 11 to the right and 04 to the left and in 01 case $(1,56 \%)$ to the left, it'd origin three main branches. When in an only trunk, your trajectory went descending of medial for lateral in relationship to the lateral margin of the rectus abdominal muscle. When forked, your branches separated one of the other immediatelly after your emergency, back converging close to the umbilical scar. 


\section{REFERENCIAS BIBLIOGRÁFICAS}

Arnold, M. The surgical anatomy of the blood supply. J. Thorac. Cardiovasc. Surg., 64:596, 1972.

Basmajian, J. V. Parede anterior do abdome e escroto. In Anatomia de Grant. 10ª ed. São Paulo, Manole, 1980. p143.

Boyd, J. B.; Taylor, G. I. \& Corlett, R. The vascular territories of the superior epigastric and the deep inferior epigastric systems. Plast. Reconst. Surg., 73:2-16, 1984.

Briand, E.; Gerbault, O.; Revol, M. et al. Abdominal wall: flaps donor site. Theoretical basis, blood supply and practical consequences. Ann. Chir. Plast. Esther., 44:40110, 1999.

Castro, S. V. Anatomia fundamental. $2^{\mathrm{a}}$ ed. São Paulo, Mc Branw-Hill do Brasil, 1985. p161.

Dangelo, J. G. \& Fattini, C. A. Anatomia Humana sistêmica e segmentar. 2a ed. Rio de Janeiro, Atheneu, 1998. p504.

Elbaz, J. S.; Dardour, J. C. \& Ricbourg, B. Vascularisation artérielle da la paroi abdominalle. Ann. Chir. Plast., 20:19-29, 1975.

Gardner, E.; Gray, D. J.; O'rahilly, R. O abdomen. In Paredes abdominais. $4^{\mathrm{a}}$ ed. Rio de Janeiro, Guanabara Koogan, 1982. p352.

Gerard, G. Angiologie. In Manuel d'anatomie humaine. Paris, Steinheil Éditeur, 1912. p449.

Gray, H. \& Goss, C. M. Anatomia. 29a ed. Rio de Janeiro, Guanabara Koogan, 1988. p501.

Haddad, C. M. Ensaios sobre a rede arterial da parede antero - lateral do abdome: Estudo radiológico. Rev. Assoc. Med. Bras., 10(14):255-8, 1968.

Hollinshead, W. D. Livro texto de anatomia humana. São Paulo, Harper \& Row do Brasil, 1966. p566.

Hurd, W. W.; Bude, R. O. ; Delancy, J. O. L \& Newman, J.S. The location of abdominal wall vessels in relationship to abdominal landmarks apparent at laparoscopic. Am. J. Obstet. Gynecol., 171(3):642-6, 1994.

Latarjet, M.; Ruiz-Liard, A.R. Circulação sistêmica: Artéria subclávia. In Anatomia Humana. $2^{\mathrm{a}}$ ed. São Paulo, Pan Americana, 1993. p1113.
Lindner, H. H. The anterolateral abdominal wall. In Clinical anatomy. California, Appleton \& Lange, 1989. p299.

Meunier, B.; Watier, E.; Leveque, J.; Roche, G.; Rolland, Y. \& Pailheret, J. P. Pre-operatore color-Doppler assessment of vascularisation of the rectus abdominis: Anatomic basis of breast reconstruction with a transverso and rectus abdominis myocutaneo flap. A prospective study. Surg. Radiol. Anat., 19:35-40, 1997.

Milloy, F. J.; Anson, B. J. \& Mcafee, D. K. The rectus abdominis muscle and the epigastric arteries. Surg. Gynecol. Obstet., 110:239, 1960.

Moon, H. K. \& Taylor, G. I. The vascular anatomy of rectus abdominis musculocutaneus flap based on the deep superior epigastric system. Plast. Reconstr. Surg. 5(82):815-32, 1988.

Moore, K. L. O abdome. In Anatomia orientada para a clínica. $3^{\text {a }}$ ed. Rio de Janeiro, Guanabara Koogan, 1992. p112.

Nahai, F.; Brown, R. G. \& Vasconez, L. Blood supply to the abdominal wall as related to planning abdominal incisions. Am. Surg., 9(42):691-5, 1976.

O’dey, D. M.; Heimburg, D.V.; Prescher, A. \& Pallua, N. The arterial vascularisation of the abdominal wall with special regard to the umbilicus. $\mathrm{B} r$. J. Plast. Surg., 57(5):392-7, 2004.

Orts-llorca, F. O. Aparato locomotor. Parte descriptive. Abdome. In Anatomía humana. $4^{\mathrm{a}}$ ed. Madrid, Científico-Médico, 1970. p614.

Paturet, G. Myologie. In: Traité d'anatomie humane. Paris, Masson \& Cie Éditeurs, 1951. p923.

Rocha, A. C. Biometria e sintopia da artéria epigástrica superior [Tese]. São Paulo, Universidade Federal de São Paulo, 2005.

Romanes, G. J. Cunningham Manual de anatomia prática. São Paulo, Atheneu. 1976. p136-137.

Rouviére, H. Artéres de la tête et du cou. In: Anatomie humaine. Descriptive et topographique. 50a ed. Paris, Masson et Cie, Éditeurs, 1943. p206. 
Rubinstein, E. Vascularização da parede abdominal. In: Petroianu, A. Anatomia cirúrgica. Rio de Janeiro, Guanabara Koogan, 1999. p376.

Schaefer, J. P. Angiologia. In: Morris'. Human anatomy. $7^{\text {a }}$ ed. New York, McGraw-Hill, 1989. p673.

Snell, R. S. O abdome: A parede abdominal. In: Anatomia clínica para estudantes de medicina. $5^{\mathrm{a}}$ ed. Rio de Janeiro, Guanabara Koogan, 1995. p140.

Tandler, J. Angiologia. In: Tratado de anatomia sistemática. Barcelona, Salvat Editores, 1929. p155-6.

Testut, L. \& Latarjet, A. Músculos del abdome. In: Compendio de anatomia descriptiva. $17^{\mathrm{a}}$ ed. Barcelona, Hispano América, 1959. p921-5.

Voss, H. \& Herrlinger, R. Anatomia humana. Buenos Aires, Atheneu, 1964. p276.
Dirección para correspondencias: Prof. Amauti Clemente da Rocha Disciplina de Anatomía Descriptiva e Topográfica Universidad de Ciencias de la Salud de Alagoas.

Rua Jorge de Lima, 113 - Trapiche

CEP: $57010-300$

Maceio-Alagoas

BRASIL

Email:amauri.rocha@uol.com.br

Recibido : 14-09-2005

Aceptado: 25-11-2005 